\title{
Pandemia, envejecimiento y políticas públicas en América Latina. Apuntes teóricos para pensar el problema de las vejeces desiguales desde los enfoques del curso de vida y de la economía política del envejecimiento
}

\section{Sofía Clarisa Marzioni}

(iD) https://orcid.org/0000-0001-5373-4057

Consejo Nacional de Investigaciones Científicas y Técnicas (CONICET), Argentina sofimarzioni@hotmail.com

RESUMEN

La pandemia causada por el COVID-19 puso de relieve la diversidad y la desigualdad existente entre las personas mayores en los países de América Latina. ¿Desde qué marcos teóricos es posible emprender el análisis de las vejeces desiguales? ¿Cuál es el rol que juegan el Estado y las políticas públicas y sociales? Este artículo surge de la convicción de que es necesario revisar los marcos analiticos que se utilizan para entender el envejecimiento. Se propone problematizar las vejeces desiguales a partir de repasar y articular algunas premisas teóricas de dos enfoques: el del curso de vida y el de la economía política del envejecimiento. Se argumenta que estos permiten captar mejor la complejidad de la realidad social, nutriendo diagnósticos más certeros sobre la situación y los problemas de las personas mayores en la región, que pueden servir como punto de partida para el diseño de nuevas políticas y para interpelar las potencialidades y límites de las ya existentes.

Palabras clave: envejecimiento, vejeces desiguales, politicas públicas y sociales. 


\section{Pandemic, aging and public policies in Latin America. Theoretical notes to think about the problem of unequal old age from the perspec- tives of the life course and the political economy of aging}

\section{ABSTRACT}

The pandemic caused by the COVID-19 highlighted the diversity and inequality among older people in Latin American countries. From what theoretical frameworks is it possible to undertake its analysis? What is the role played by the State and public and social policies? This article arises from the conviction that it is necessary to review the analytical frameworks used to understand aging. It is proposed to problematize unequal old age by reviewing and articulating some theoretical premises of two approaches, that of the life course and that of the political economy of aging. It is argued that these allow a better grasp of the complexity of social reality, nurturing more accurate diagnoses about the situation and problems of the elderly in the region, which can serve as a starting point for the design of new policies and to question the potentialities and limits of existing ones.

Keywords: Aging, unequal old age, public and social policies. 


\section{PRESENTACIÓN 12}

Este artículo profundiza sobre el problema de la desigualdad existente entre las personas mayores en América Latina, asunto que reviste sustantivo interés en el contexto pandémico, cuando las diferencias se hacen evidentes y, aún más, se profundizan. La revisión del estado de las investigaciones en la materia permite identificar algunos puntos salientes en el recorrido trazado por los estudios del envejecimiento. Durante mucho tiempo, primó sobre este una mirada reduccionista, deudora de las primeras teorías en el campo que estuvieron enmarcadas en perspectivas biológicas y médicas. En estas interpretaciones, el envejecimiento fue entendido como un proceso homogéneo y la vejez se caracterizó como una etapa de la vida estancada, pasiva, de pérdidas y deterioro. Los primeros abordajes de la gerontología tampoco dieron a la heterogeneidad del envejecimiento la importancia debida y la consideraron como resultado de la voluntad o la iniciativa individual. Así, le quitaron relevancia a la desigualdad como problema teórico, cuya inclusión definitiva en los trabajos científicos tardó en producirse (Oddone, 2018).

Desde hace algunas décadas, el envejecimiento sociodemográfico se hizo evidente en gran parte de los países del mundo (DESA population, 2019; Lesson, 2013). El crecimiento de la proporción de personas mayores en el total de la población abrió múltiples interrogantes que impulsaron la investigación científica en el campo gerontológico, adquiriendo esta gran dinamismo y aumentando la

1 Este artículo se enmarca en la investigación (en curso) «Políticas sociales y ciudadanía en la vejez. Un análisis desde las políticas, las biografías y las subjetividades en la ciudad de Santa Fe, Argentina», llevada adelante con el apoyo de una beca del Consejo Nacional de Investigaciones Científicas y Técnicas de Argentina para la obtención del doctorado en Ciencia Política, Universidad Nacional de Rosario, Argentina.

2 Para aligerar la lectura del escrito se utiliza una redacción convencional, sin perjuicio de la política de no discriminación de géneros, a la cual la autora adhiere. 
participación en este debate de las ciencias sociales ${ }^{3}$. Aunque las interpretaciones estereotipadas sobre la vejez se extendieron rápidamente al envejecimiento poblacional —entendido mayormente como un «problema» [sic] — (Pérez Díaz, 2016), también surgieron esfuerzos en otros sentidos. Actualmente se asiste, entonces, al desarrollo de nuevas miradas que ponen de manifiesto la diversidad como rasgo sustantivo del envejecer y problematizan la desigualdad existente entre las personas mayores, llamando la atención sobre sus causas estructurales (Iacub, 2002; Oddone, 2018; Yuni, 2019). Tales contribuciones, se sostienen, además, sobre posturas epistemológicas (y políticas) que enfatizan la condición de las personas mayores como «sujetos de derecho» y reivindican sus aportes a las comunidades de las que forman parte.

Inmersos en esa gerontología crítica, los enfoques del curso de vida y de la economía política del envejecimiento han hecho lo propio. En los apartados que siguen se revisarán algunas de sus premisas teóricas y se las articulará en un marco explicativo general sobre la desigualdad que aqueja a las personas mayores en América Latina, tomando la pandemia como coyuntura de análisis. Se sostendrá aquí que entender este estado de cosas requiere centrarse en el curso de vida de las personas, en tanto los ciudadanos de mayor edad «arrastran» hacia la vejez una posición en la estructura social que se va forjando desde los primeros momentos de sus existencias. Esa posición no constituye un reflejo lineal del «mérito» de cada quien, sino que es resultado también de la injerencia de factores socioestructurales, en cuya configuración el Estado y sus políticas públicas y sociales ocupan un rol central (Bengston, Elder y Putney, 2005; Dannefer, 2003; Dannefer y Settersten, 2010; O' Rand, 2006; Walker, 2009, entre otros). Este argumento puede no resultar novedoso a todos los lectores, pero es menester reconocer que gran parte de la investigación gerontológica solo hace referencias pasajeras a estos tópicos, aun en los casos en que se manifiesta una preocupación explícita por la desigualdad (Kending y Nazroo, 2016). Vale la pena, entonces, considerar este problema con mayor detenimiento y, al mismo tiempo, aportar a la «traducción» de estas perspectivas al idioma español y a la realidad latinoamericana, puesto que, en su mayoría, se han desarrollado en otros contextos.

Conviene, por último, advertir que, aunque el presente es un trabajo de corte teórico, el interés por su desarrollo no es meramente cognoscitivo, sino también

En diferentes disciplinas, se fueron desarrollando nuevos campos de estudios, como la «antropología de las edades» (Feixa, 1996) o la «gerontología política» (Ares Castro Conde, 2018), para mencionar algunas. 
político. Se inserta en una búsqueda por contribuir a comprender la realidad social circundante en orden a obtener mejores diagnósticos sobre los problemas sociales que permitan repensar el quehacer del Estado. En este sentido, vale mencionar que, si las vejeces desiguales son un asunto ampliamente aceptado en la gerontología, las políticas públicas y sociales están «descubriendo» la necesidad de revisar su orientación que, tradicionalmente, ha girado en torno a las personas mayores comprendidas como una población homogénea.

\section{ELPROBLEMA DE LAS VEJECES DESIGUALES EN AMÉRICA LATINA Y EL CONTEXTO PANDÉMICO}

El envejecer de las personas es, en sí mismo, un proceso «diferencial» (Knopoff y Oddone, 1991). Como se explica desde la gerontología,

[...] la vejez se configura como una construcción socio-cultural, sobredeterminada por dimensiones contextuales socio-económico-político-culturales que atraviesan la vida cotidiana; de allí que el envejecer sea un proceso particular y complejo que comprende diferentes aspectos: físico-biológicopsicológico-social y emocional, constituyéndose en una experiencia única en relación a estos aspectos y dimensiones (Ludi et al., 2018, p. 58).

En consecuencia, aunque culturalmente suele concebirse el envejecimiento desde representaciones homogéneas — propias de las formas en que originalmente este fue descripto desde las disciplinas biológicas y médicas-, se observan más diferencias que similitudes en los modos de envejecer de personas con disímiles características físicas o psicológicas, pertenecientes a distintas clases sociales, géneros o etnias, radicadas en zonas rulares o urbanas, entre otras condiciones (Oddone, 2014). De acuerdo con la propuesta de Lalive d'Espinay (1999), es útil hablar de «vejeces» (en lugar de «vejez») para dar cuenta de esta pluralidad (Ludi et al., 2018).

Atendiendo a las especificidades del envejecimiento en América Latina, es posible sumar como complementaria la categoría de «vejeces desiguales», que resulta provechosa para examinar la vinculación de la cuestión social con las condiciones de desigualdad en la vejez, teniendo en mente los procesos sociohistóricos regionales y nacionales (Manes et al., 2016). Esta mirada implica cuestionar la categoría de vejez como única, retomando la perspectiva del envejecimiento diferencial y agregando la categoría de desigualdad para dar cuenta de las condiciones de explotación y vulneración de los mayores en la región 
(Manes, Garmendia y Danel, 2020). De hecho, son muchas las personas mayores que están condenadas a sobrevivir, sin poder disfrutar de los «nuevos» años que permiten alcanzar los avances médicos y tecnológicos ni proyectar cómo desean transitar este momento de su vida (Ludi, 2005). En este sentido, es importante advertir que circunstancias elementales para el desarrollo de condiciones de vida mínimas, como el acceso a los servicios básicos, continúan siendo una problemática en la región: aproximadamente un 13\% de los hogares no tiene acceso a fuentes de agua mejoradas, situación que en las zonas rurales alcanza al 25\% de los hogares (Cepal, 2020c). Así las cosas, el envejecimiento de la población se produce en estas latitudes a un ritmo acelerado y en un entorno caracterizado por la pobreza, la informalidad laboral, los problemas en el acceso a los servicios de salud y una escasa cobertura o calidad de la seguridad social, como se viene señalando desde la Primera Conferencia Regional Intergubernamental sobre el Envejecimiento realizada en el año 2003 (Cepal, 2003)

El ya desafiante escenario terminó por completarse con la irrupción de la pandemia. Hacia fines del año 2019, la Organización Mundial de la Salud reportó los primeros casos de COVID-19, detectados en Wuhan, China. Como consecuencia de un mundo cada vez mejor interconectado, el virus se esparció a una velocidad inusitada por diferentes países. Ingresó al continente americano hacia enero del año 2020, creando una crisis sociosanitaria que mantiene en vilo a sus gobiernos y ciudadanos. Si bien se trata de un fenómeno global, interesa destacar que, en el ámbito regional, agrava el panorama económico y social: se estima una contracción económica del 9,1\%, con aumentos significativos de la tasa de pobreza, que alcanzará el $37,3 \%$ e incrementos de la tasa de desocupación que llegará al 13,5\%. Como resultado, la desigualdad — manifiesta en las clases sociales, los géneros, las edades o los territorios, entre otras dimensiones-recrudece en América Latina, que sigue ratificándose como la región más desigual del mundo (Cepal, 2020b). La recesión económica y otras consecuencias más amplias del COVID-19 impactan en la percepción de ingresos de las personas mayores: sus oportunidades de trabajo se vuelven más limitadas — sobre todo en el caso de las mujeres y las personas con discapacidad - y los montos de sus pensiones resultan aún más inadecuados ante la magnitud de las necesidades que el implica el día a día en este nuevo escenario. A la vez, los sistemas de pensiones — que ya registraban bajos niveles de protección social y problemas

4 Para profundizar sobre este punto puede consultarse p.ej.: HelpAge-international, 2019; Huenchuan, 2018; OISS e IMSERSO, 2006. 
de financiamiento - son afectados por la pandemia mediante el descenso de la cotización, especialmente en los países con mayores niveles de desempleo e informalidad (Cepal, 2020a).

Al mismo tiempo, en pleno crecimiento del envejecimiento de la población a escala mundial, el COVID-19 llama la atención sobre las brechas en las oportunidades y condiciones de vida de las personas mayores en la región, en tanto la enfermedad y la crisis no afectan del mismo modo ni con iguales consecuencias a todos los ciudadanos de mayor edad. Se descubren, de hecho, realidades disímiles en el contexto pandémico al comparar situaciones nacionales o al interior de un mismo país entre diferentes grupos de personas mayores. Los contrastes se vuelven evidentes ante las diferentes posibilidades de resguardarse, de sobrevivir ante el eventual contagio o de acceder a la vacunación y a los cuidados médicos y sociales, por poner algunos ejemplos. El acceso diferencial a los servicios de agua y saneamiento, cruciales para la vida, la salud y la higiene, expresa una de las consecuencias más críticas de la desigualdad y cómo esta juega en la contención de la enfermedad ${ }^{5}$. Desde los organismos internacionales de derechos humanos, se alertó que resulta especialmente preocupante la situación de aquellas personas mayores que padecen patologías previas, viven en la pobreza, no disponen de acceso a servicios de salud de calidad y se encuentran en soledad o en espacios confinados, tales como cárceles o residencias (Kornfeld-Matte, 2020). Todavía más preocupantes son los casos en que una misma persona acumula dos o más de estas desventajas; por ejemplo, alguien podría tener una enfermedad crónica, no tener ingresos suficientes ni contar con apoyo familiar.

Respecto de las políticas de cuidado - especialmente implicadas en el combate contra el COVID-19-, la pandemia ha puesto de relieve la poca visibilidad y desarrollo del sector: salvo algunas excepciones, no existe aún en los países de la región una oferta pública y estatal unificada ni con amplia cobertura. Mientras este tema se abre paso en las agendas públicas y de gobierno, el cuidado diario de las personas mayores se resuelve mayoritariamente en el ámbito familiar, recayendo entre las responsabilidades asumidas tradicionalmente por las mujeres dentro de los hogares. Ese intenso nivel de familiarización de los cuidados se corresponde no solo con una oferta estatal insuficiente, sino también con un alto costo de los

5 De acuerdo con una revisión de datos censales realizada por la Cepal, los porcentajes de personas mayores que viven en hogares sin acceso a agua varían entre los diferentes países: puede ir desde un $4 \%$ en Chile a un $16 \%$ en Guatemala. La situación es más preocupante en cuanto al acceso a servicios de saneamiento adecuado para las viviendas. En los dos casos, el problema se agrava en las zonas rurales (Cepal, 2020a). 
servicios mercantilizados de calidad, que solo están disponibles para el sector de la población con mejores ingresos y una oferta comunitaria limitada, aunque no por eso desdeñable. La pandemia tensiona abiertamente esta organización social del cuidado, demostrando la necesidad de reconocer la importancia de los cuidados para la sostenibilidad de la vida y de legitimar al sector de los cuidados como un componente fundamental para el desarrollo de los países de la región (CEPAL, 2020c).

Todo lo dicho crea un estado de cosas que desafía a las políticas públicas y sociales, entendidas como aquellas acciones del Estado dirigidas - al menos, en el discurso gubernamental explícito- a mejorar las condiciones de vida y promover la igualdad entre los ciudadanos (Adelantado et al., 2000). Vale considerar que tales políticas encuentran una guía rectora en los instrumentos de derechos humanos, las cuales ostentan su forma más acabada en la Convención Interamericana sobre la Protección de los Derechos Humanos de las Personas Mayores, adoptada durante la 45 a Asamblea General de la OEA de $2015^{6}$. De características únicas en el mundo, este tratado prohíbe la discriminación y reconoce a las personas mayores los derechos al bienestar, la salud y los cuidados (Dabove, Oddone, Perret y Pochintesta, 2020). En esta línea, los organismos internacionales y las organizaciones de defensa de los derechos de las personas mayores han venido emitiendo recomendaciones de políticas para la protección de las personas mayores frente al COVID-197.

Los párrafos precedentes advierten sobre la complejidad que reviste la realidad social de las personas mayores en América Latina y en el contexto de la pandemia causada por el COVID-19. Resulta evidente la necesidad de superar la visión tradicional del envejecimiento como un objeto de conocimiento universal para, en su lugar, poder desentrañar la pluralidad de situaciones, vinculadas con estas vejeces desiguales, que requieren de una atención más atenta por parte del Estado. Ahora bien, ¿a qué se debe este estado de cosas? ¿Dónde se encuentran las causas de la desigualdad entre las personas mayores? ¿Cuál es el rol que juegan el Estado y las políticas públicas y sociales con relación a ellas?

\footnotetext{
6 Vale considerar que a la fecha esta se encuentra ratificada en Argentina, Chile, Ecuador, El Salvador y Perú. Recuperado de http://www.oas.org/es/sla/ddi/tratados_multilaterales_ interamericanos_A-70_derechos_humanos_personas_mayores_firmas.asp, información consultada en mayo de 2021 .

7 Dentro de este conjunto de publicaciones, pueden consultarse Cepal (2020). COVID-19, Recomendaciones generales para la atención a personas mayores desde una perspectiva de derechos humanos; CISS (2020). Adultos mayores y COVID-19: algunas recomendaciones urgentes para salvaguardar a los más vulnerables. CISS; HELPAGE (2020). Pautas para residencias de personas mayores en el contexto de corona virus (COVID-19). Londres: HelpAge.
} 


\section{ENFOQUES TEÓRICOS PARA (RE)PENSAR EL ENVEJECIMIENTO: EL CURSO DE VIDA Y LA ECONOMÍA POLÍTICA}

Con el objetivo de proponer algunas respuestas a los interrogantes recién mencionados, en este apartado y los que siguen se revisarán y articularán algunas premisas de dos enfoques teóricos: el curso de vida (en adelante CV) y la economía política del envejecimiento (en adelante EPE).

En un esfuerzo por sintetizar sus principales características, el primero de estos enfoques puede ser definido como sigue:

El estudio interdisciplinario del desarrollo de la vida humana (ontogénesis humana), mediante el establecimiento de puentes conceptuales entre (a) los procesos de desarrollo biológicos y psicológicos; (b) el curso de la vida como institución social, desde el doble punto de vista: el de las regulaciones sociales y culturales de la cual es objeto y de su construcción por los individuos en función de sus recursos y el de sus perspectivas biográficas; (c) el contexto socio histórico y los cambios que este experimenta (Lalive D’Epinay et al., 2011, p. 20).

Como deja ver la cita, el enfoque del CV implica un cambio de perspectiva en el sistema de creencias sobre la vida humana, asociado con la institucionalización de nuevos modelos de ciencia. Su surgimiento fue posible en el contexto de una transformación epistémica más amplia, que tuvo implicancias en el estudio del envejecimiento, configurándose en su marco un movimiento crítico a la gerontología tradicional (Yuni, 2011). En ese clima, diferentes líneas de investigación (los trabajos demográficos sobre cohortes y generaciones, la psicología del desarrollo, llamada life span, la sociología que estudia la articulación entre la historia y las vidas individuales y la antropología que estudia cómo el desarrollo de la existencia es codificado socioculturalmente) convergieron y dieron origen al «paradigma del curso de la vida» (Lalive D'Epinay et al., 2011).

¿Cuál es el legado del enfoque del CV con relación al estudio del envejecimiento de las personas? Este revela que la ontogénesis humana es un fenómeno multidimensional y, por lo tanto, debe ser estudiado en forma interdisciplinaria $\mathrm{y}$, al mismo tiempo, que dicho desarrollo se produce a lo largo de toda la existencia de la persona, desde el nacimiento hasta su muerte, experimentándose de diferentes maneras según cada caso. De aquí deriva la idea de que «envejecemos a partir del momento en que nacemos» y de la diversidad como característica sustantiva de dicho proceso» (Gastrón y Oddone, 2008). De este modo, supera la visión de la gerontología tradicional del desarrollo humano compuesto por ciclos 
o estaciones de la naturaleza, reemplazándola por la idea de curso «que posee una connotación de recorrido, apertura, continuidad, trayectoria e indeterminación [...] consistente con el cambio de la cosmovisión moderna acerca del tiempo y la temporalidad en tiempo de posmodernismo» (Yuni, 2011, p. 133). Asimismo, el concepto de ciclo de vida supone una definición más limitada, que se centra en cambios específicos, mientras que el de curso de vida pone énfasis en cómo esos cambios se relacionan con el entorno, dando una visión más compleja sobre el desarrollo humano (OPS y OMS, 2021).

Por otra parte, el enfoque del CV identifica y describe las diferentes etapas de la vida (infancia, juventud y vejez) y los pasajes de una a otra, observando a estas como objeto de trabajo de la sociedad y la cultura. Lo hace poniendo de relieve que las ganancias y pérdidas se reequilibran de manera continua a lo largo de la vida y que el envejecimiento conlleva beneficios y potencialidades que pueden expresarse tanto en el ámbito individual como en el colectivo. En consecuencia, refuta la representación de la vejez como un proceso unidimensional de pérdidas, que se justifica sobre interpretaciones edadístas (Yuni, 2011). También permite comprender que las vejeces son una construcción social e individual, en tanto están limitadas por las pautas sociales y culturales: expectativas de rol y normas de la edad, que ciñen lo que «debe ser y hacer» una persona mayor y, a la vez, por las experiencias que cada persona atraviesa a lo largo de su existencia (Gastrón, Oddone y Lynch, 2011).

Para este trabajo, uno de los aspectos a destacar - especialmente del enfoque del CV- es su interés por desentrañar el modo en que se entrecruzan la dinámica de los cambios y eventos propios de una sociedad determinada - como puede ser la pandemia causada por el COVID-19- y las trayectorias de vida de los individuos que la componen. Hay, entonces, dos tipos de temporalidades relevantes: por una parte, la de la vida humana $\mathrm{y}$, por otra, la de la vida en sociedad. Al articularlos, el individuo aparece no ya en su vida aislada sino en su inserción sociohistórica y colectiva (Lalive D’Epinay et al., 2011). A modo de ejemplo, pueden considerase las investigaciones de Elder —entre las cuales destaca la publicada bajo el título Children of the Great Depression (1974)-, que dan cuenta de la complejidad del cambio social y su influencia sobre las vidas de las personas, así como también, a la inversa, de las formas en que estas pueden influir en el ámbito social. En suma, el enfoque del CV tiene la virtud de introducir una perspectiva temporal longitudinal y sociohistórica en campos que, hasta el momento, habían sido concebidos a partir de perspectivas transversales (Lynch, 2015). 
Por su parte, el enfoque de la EPE emerge hacia la década de 1980 y se instala como uno de los más importantes en la gerontología actual, poniendo en diálogo el estudio del envejecimiento con el de las teorías de la estratificación social y las teorías del Estado y de las políticas públicas y sociales (Bengtson et al., 2009). Se orienta a examinar el modo en que las estructuras sociales inciden en la forma en que las personas transitan la vejez. Pone el foco de atención en el rol de las instituciones y las políticas públicas respecto de la posición social que ocupan los ciudadanos de mayor edad dentro del espacio político del Estado-nación. Consiguientemente, «su forma de pensar es sistémica y fundada en el principio de que la vejez puede ser entendida solamente por el estudio de los problemas y asuntos del orden social mayor» (Sánchez, 2005, p. 106, citado en Piña, 2010).

Este segundo enfoque también participa de la gerontología crítica. Más precisamente, puede ser considerado como parte de una generación de teorías sobre el envejecimiento que tienen la vocación de vincular el estudio de los aspectos micro y macrosociales. Así, los representantes de la EPE, entre los que se destaca Estes, entienden como uno de los principales desafíos de la gerontología la necesidad de comprender cómo las personas interpretan sus problemas individuales, pero también - y fundamentalmente - cómo estos son convertidos en cuestiones de interés colectivo y objeto de políticas públicas (Díaz Tendero Bollain, 2016).

Una revisión de las investigaciones realizadas desde el enfoque de la EPE deja ver dos grandes momentos en cuanto a los temas de interés y de estudio. Una primera ola de contribuciones se especializa en los estados de bienestar y en su relación con las personas mayores. Aquí se pueden situar la clásica tipología de Esping-Andersen (1989) y las consiguientes revisiones y críticas provenientes de los estudios feministas y étnicos. Una segunda ola de trabajos atiende al momento de las reformas de los estados de bienestar y sus consecuencias para el grupo poblacional de mayor edad. Se perfilan dos discursos opuestos: el de la equidad generacional — esgrimido en favor de la solidaridad social—y el de la interdependencia generacional, que promociona la privatización de los sistemas de pensiones (Díaz Tendero Bollain, 2016).

Entre los principios teóricos más relevantes del enfoque de la EPE, se cuentan, por un lado, aquellos que plantean que los factores sociales, políticos y económicos influyen en la definición y el abordaje de los problemas sociales asociados a las personas mayores; a la vez que tales definiciones y abordajes, que se materializan en políticas públicas y sociales, repercuten en la situación y posición de la población envejecida (Piña, 2010). Por otro lado, están aquellos que enfatizan que las 
oportunidades vitales y los recursos de las personas mayores están dados por la posición que estas ocupan en la estructura social (Díaz Tendero Bollain, 2016).

El enfoque de la EPE tiene la virtud de dar cuenta de la variabilidad intracohorte existente entre las personas mayores, ya que toma en consideración diferentes ejes generadores de estratificación, como son la clase, el género o la etnia. Por lo demás, se destaca en esta construcción teórica una interpretación ampliada de los problemas de la vejez que son puestos en relación con el contexto social, incluyendo como parte central al Estado y a sus acciones, principalmente aquellas en materia de seguridad y asistencia social ( Piña, 2010).

\section{LAS VENTAJAS Y DESVENTAJAS ACUMULATIVAS EN EL CURSO DE VIDA COMO EXPLICACIÓN DE LAS VEJECES DESIGUALES}

Como se ha señalado más arriba, desde el $\mathrm{CV}$ se considera que las experiencias tempranas impactan en la vida posterior de la persona, observándose la interacción de estas con las estructuras sociales (Kending y Nazroo, 2016). Puesta la atención en el curso de vida, es el concepto de «ventajas y desventajas acumulativas» (Dannefer, 2003) el que habilita a captar el proceso por el cual se incrementa la desigualdad entre individuos de una misma cohorte respecto de una determinada característica, como puede ser el ingreso, la salud, las condiciones laborales, entre otras (Bengston, Elder y Putney, 2005; Dannefer y Settersten, 2010; O’Rand, 2006; Walker, 2009, entre otros). Dicho de la manera más simple posible, aquellas personas que tienen más ventajas se vuelven todavía más aventajadas con el paso del tiempo, y aquellas que tienen más desventajas se vuelven todavía más desventajadas con el paso del tiempo.

El concepto de ventajas y desventajas acumulativas fue acuñado por Robert Merton en 1968 en su trabajo sobre la estratificación de las carreras científicas, The Matthew Effect in Science. En este sostuvo que, dentro de una misma cohorte de científicos, las desigualdades tienden a incrementarse en el curso de sus trayectorias al enlazarse con desigualdades previas (Dannefer, 2003; O'Rand, 2006). Este aporte sirvió como disparador para el estudio de la heterogeneidad y desigualdad intracohorte que se desarrolla a lo largo del curso de vida y que se manifiesta más claramente en la vejez (Saraví, 2020). Al ser la edad y la acumulación dos fenómenos inherentemente temporales, resultó lógica su articulación posterior en los estudios sobre el envejecimiento (Dannefer, 2003).

La idea de que las vejeces desiguales pueden ser mejor comprendidas en función de las ventajas o desventajas que se encadenan en cada devenir individual merece algunas precisiones conceptuales: 
La ventaja / desventaja acumulativa puede ser definida como la tendencia sistémica a la divergencia interindividual en una característica dada (por ejemplo, dinero, salud o estado) con el paso del tiempo. Dos términos de esta definición requieren atención: La «tendencia sistémica», indica que la divergencia no es una simple extrapolación de las posiciones respectivas de los miembros en el punto de origen; resulta de la interacción de un complejo de fuerzas. Mientras que, la «divergencia interindividual», implica que la ventaja/ desventaja acumulativa no es una propiedad de individuos sino de poblaciones u otras colectividades (como cohortes) (Dannefer, 2003, p. 327) ${ }^{8}$.

Saraví (2020) se detiene a establecer algunos matices en torno a este concepto, brindando una interpretación más detallada sobre los mecanismos por los cuales puede operar la desigualdad. Para tal fin, diferencia entre incremento o encadenamiento, acumulación diacrónica y sincrónica y desventaja acumulativa o acumulación de desventaja. Sin embargo, estos diferentes procesos redundan en un mismo resultado: una dispar distribución de oportunidades y recursos que resulta, en definitiva, en modos diversos de envejecer.

Esta perspectiva fue adoptada inicialmente por la sociodemografía norteamericana y sus precursores fueron autores como Dannefer y O'Rand'. Sin embargo, es recomendable su aplicación a otros contextos. Pensar en términos de ventajas y desventajas acumulativas resulta especialmente útil para estudiar el envejecimiento en América Latina, en tanto ello nos habilita a comprender la pobreza y la desigualdad como procesos en los que se articulan múltiples dimensiones durante la trayectoria biográfica (Bayón, 2015). En efecto, una línea vigente de investigaciones viene operacionalizando el estudio de la exclusión social como un fenómeno de acumulación de desventajas, aunque sin prestar especial atención a la vejez (Saraví, 2020) ${ }^{10}$. Puede ser provechosa también la perspectiva comparativa entre países, que arroja luz sobre las diferencias entre estos, permitiendo identificar con mayor claridad las influencias estructurales y las variaciones de políticas en cada caso - más difíciles de reconocer en los estudios sobre los individuos dentro de un conjunto de estructuras sociales- (Kending y Nazroo, 2016).

La traducción es nuestra.

Para profundizar sobre el tratamiento de las ventajas y las desventajas acumulativas desde el enfoque del CV, ver Dannefer (2003).

10 Para una síntesis de la producción en América Latina sobre la acumulación de ventajas y desventajas ver Saraví (2020). 


\section{¿DESIGUALDAD DE MÉRITOS O DESIGUALDAD SOCIOESTRUCTURAL? ZANJANDO EL DEBATE}

Como apuntó inicialmente Merton (1968) al investigar las carreras científicas, indagar en las ventajas y desventajas acumulativas explica la existencia y las fuentes de diferenciación en el tiempo, dando cuenta de que estas ocurren independientemente del mérito (Saraví, 2020). Aplicar este concepto al estudio del envejecimiento habilita, pues, a reconocer los factores socioestructurales que moldean el envejecer a lo largo del curso de vida. Ello significa que la desigualdad entre las personas mayores no es principalmente el resultado de decisiones y acciones individuales, tampoco de características genéticas o talentos naturales, sino que, en lo fundamental, se debe a factores socioestructurales (Ferraro y Shipee, 2009; Walker, 2009).

Ahora bien, si la desigualdad entre las personas mayores es socioestructural, ¿qué factores la generan? Walker (2009) sintetiza los aportes en este campo de estudios y propone una respuesta a tal pregunta, identificando los siguientes: clase social de origen, educación, historia ocupacional, clase socioeconómica, género, estado de pareja, salud, etnia y edad como factores que influyen especialmente a lo largo de la vida en la determinación de las desigualdades entre las personas mayores. Nótese que la edad en sí misma es uno de los factores causantes de la desigualdad. De hecho, esta:

subyace como uno de los determinantes de la organización de instituciones sociales como la familia y de las estructuras que organizan la educación y el trabajo. Por esta vía, es un determinante de la distribución del bienestar, del poder y de la autoridad en la estructura social, así como una de las bases de la organización social en torno a la que se asignan responsabilidades y roles (Cepal, 2016, p. 24).

De esta manera, cada momento de la vida conlleva oportunidades, desafíos y riesgos específicos. En la vejez, las desigualdades relativas a la edad se explican por una serie de eventos que resultan más probables en la vida posterior, como la viudez, la fragilidad y el agotamiento de los activos. Asimismo, durante la mediana edad y la vejez, los trabajadores a menudo se ven discriminados en el empleo, en términos de promoción o acceso a la formación o, incluso, en la contratación Walker, 2009). En efecto, la discriminación por edad (o «viejismo») (Butler, 1969), al igual que aquella por clase, raza o sexo, conlleva consecuencias en el trabajo y la jubilación y, como tal, se asocia también a los procesos de acumulación de ventajas y desventajas (Estes y Di Carlo, 2020). El viejismo 
en el ámbito laboral resulta especialmente perjudicial en la región, donde gran parte de la población mayor continúa en actividad (aproximadamente un 35,4\% de las personas mayores) (Ramos Bonilla y Tirado Ratto, 2019), y lo hace en condiciones informales y precarias (Cepal y OIT, 2018). Estudiar las trayectorias laborales desde el curso de vida aplicando un enfoque biográfico, capaz de contemplar las experiencias y la subjetividad de las personas mayores, puede ayudar a comprender cómo se juegan estas prácticas discriminatorias en la cotidianeidad y cómo son significadas por las propias personas mayores (Bruno y Acevedo Alemán, 2016).

En América Latina, algunas investigaciones sumaron a los anteriores como factor estructurante de la desigualdad al territorio o el lugar de residencia. A modo de ejemplo, puede considerarse la investigación de Redondo (1990) sobre las condiciones de vida de las personas mayores y su proceso de envejecimiento en el barrio La Boca, uno de los «bolsones de pobreza urbana» de Buenos Aires.

Los factores mencionados dependen en gran medida de la forma en que se organiza la sociedad en áreas clave como la escolarización, el empleo y la herencia de ingresos y la riqueza, moldeados por las políticas sociales. Entonces, la pregunta que sigue resulta obvia: ¿cuál es el rol del Estado y sus políticas públicas y sociales en relación a las vejeces desiguales?

Antes de avanzar sobre dicha cuestión, vale un comentario adicional sobre lo que podríamos llamar la dicotomía entre los determinantes sociales y la agencia humana (OPS y OMS, 2021). ¿Qué papel juegan en el argumento que se viene desarrollando la subjetividad y las acciones de las propias personas mayores en la determinación de sus experiencias y trayectorias de vida? El punto de mira de las ventajas y desventajas acumulativas no implica negar la importancia de lo individual, aunque sí atender al poder de las realidades estructurales dentro de las cuales la agencia humana opera (Dannefer, 2003). Al respecto, resultan relevantes los aportes de Ferraro y Shippee (2009), quienes, desde esta perspectiva, proponen dos axiomas sobre el problema de la agencia humana y los aspectos simbólicos relacionados con esta: (i) las trayectorias del curso de la vida están determinadas por la acumulación de riesgo, los recursos disponibles y la agencia humana (particularmente, la agencia humana juega un papel fundamental en dar forma a la probabilidad de que la adversidad social se «meta (o no) bajo la piel» (Thoits, 2006), y (ii) la percepción de las trayectorias de la vida influye en las trayectorias posteriores (las personas tienen un sentido de cómo lo están haciendo y este influye en sus acciones posteriores). 


\section{ACERCA DEL ESTADO, LAS POLÍTICAS SOCIALES Y SUS EFECTOS SOBRE LA DESIGUALDAD ENTRE LAS PERSONAS MAYORES}

Un conjunto amplio de autores ha reconocido la incidencia del Estado y de sus políticas públicas y sociales — que en la sociedad moderna han cristalizado bajo la forma de los estados de bienestar o sociales - en las oportunidades vitales y en los recursos de sus ciudadanos (Beveridge, 1942; Korpi, 1980; Korpi y Palme, 1998; Titmuss, 1974). Por poner un caso, en su clásica obra Los tres mundos del Estado de Bienestar, Esping-Andersen (1993) demostró cómo diferentes regímenes y estados de bienestar actúan sobre la estructuración de clase y el orden social, en tanto sus rasgos determinan la solidaridad social, las divisiones de clase y la diferenciación del estatus.

En la región, las investigaciones realizadas en esta línea de pensamiento pusieron de manifiesto que los sistemas de bienestar se han desarrollado de manera fragmentada, expresando ciertos límites en comparación con sus pares europeos. Un conjunto de autores se ha dedicado a caracterizar sus particularidades (Carmelo Mesa Lago, 1991; Filgueira, 1995; Barba, 2003; Martinez Franzoni, 2007 , entre otros ${ }^{11}$. El momento presente comporta, además, grandes desafíos a la acción del Estado asociados a la crisis del modelo de la sociedad industrial y sus múltiples implicancias. Una de las fundamentales es el proceso de individualización. Diferentes teorías sociológicas dan cuenta de que nos encontramos en un cambio de época - para mencionar algunas, se habla de la sociedad del conocimiento (Castells), de la sociedad del riesgo (Beck), de la sociedad líquida (Bauman y Sennett) o de la nueva cuestión social (Rosanvallone y Castel)-. En este contexto, las trayectorias vitales se vuelven más diversas, con menos lazos y vínculos, $\mathrm{y}$ el «hacerse mayor» tiene muy poco que ver en el siglo XXI con lo que era hasta el último cuarto del siglo XX (Subirats, 2016).

Los estudios realizados desde la perspectiva del bienestar social evidencian que, en la medida en que los Estados forman parte de la estructura de producción de riesgos y protecciones, pueden - o no- contribuir a dar respuesta a los problemas emergentes (Adelantado et.al., 2000). Particularmente, como ha demostrado la economía política del envejecimiento, las instituciones de seguridad y asistencia social generan efectos en el curso de vida de las personas, desembocando en una mayor o menor desigualdad social (Quadagno y Reid, 1999 y Estes, 1999, citados

11 Para una revisión de esta biblioteca, se recomienda a Paura y Zibecchi, 2020. Recuperado de https://revistas.up.edu.pe/index.php/apuntes/article/view/1262/1495, consultado en mayo de 2020. 
en Díaz Tendero Bollain, 2018). En este marco, el Estado y sus políticas públicas y sociales cobran relevancia en tanto operan como principio organizativo de los diferentes sectores o esferas de la estructura social. En efecto, la política social mantiene una relación bidireccional con la estructura social y es un reflejo de esta y de las luchas sociales y las relaciones dominantes de poder en un momento histórico determinado, pero también puede incidir sobre la desigualdad social, es decir, en el conflicto distributivo de la riqueza social y en la reproducción simbólica de las jerarquías sociales (Adelantado et al., 2000).

La esfera estatal posee, consiguientemente, un lugar central en la organización de las desigualdades sociales y su contribución es fundamental en el conflicto distributivo y en la reproducción de las jerarquías sociales. Cuando los Estados no contribuyen al bienestar social — como fue el caso en las décadas de 1980 y 1990 en América Latina - , los riesgos y responsabilidades se reenvían a las familias, las comunidades o los mercados en caso de no poder ser cubiertos, se incrementan las situaciones de vulnerabilidad para ciertos grupos sociales (Filgueira y Rossel, 2015). En este sentido, es de esperar que, las políticas de ingreso, salud, cuidado y servicios sociales que no garanticen un piso mínimo, mantengan y reproduzcan las desigualdades sociales; en tanto, reflejan las oportunidades vitales y los recursos de cada persona según su posición social previa (Quadagno y Reid, 1999 y Estes, 1999, citados en Díaz Tendero Bollain, 2018; Walker, 2006).

Por su parte, el lugar que ocupan las personas mayores en la estructura social — según cual sea su clase, género, raza/etnia, entre otras causantes de la estratificación - resulta determinante de los recursos económicos y sociales de los que esta dispone y, por ende, de su experiencia del envejecer (Díaz Tendero Bollain, 2018). Al mismo tiempo, las estructuras o los sistemas sociales dan forma al comportamiento humano y a las relaciones interpersonales (Ferraro y Shipee, 2009). A decir de Huenchuan (2004, p. 160):

La vejez puede ser una etapa de pérdidas, pero también de plenitud, dependiendo de la combinación de recursos y estructura de oportunidades individuales y generacionales a la que están expuestos los individuos en el transcurso de su vida, de acuerdo con su condición y posición dentro de la sociedad. Esto remite a conjugar la edad con otras diferencias sociales - tales como el género, la clase social o la etnicidad - que condicionan el acceso a dichos recursos y oportunidades, así como la posibilidad de disfrutarlos, todo lo cual da origen a la heterogeneidad del envejecimiento en general y de las personas mayores en particular. 
En suma, desde la articulación del enfoque del CV y de la EPE, resulta central para el entendimiento de las vejeces desiguales abordarlas como «el resultado de procesos acumulativos en el tiempo de decisiones, carencias, ventajas, eventos y experiencias que se articulan, además, con los arreglos institucionales, situados en contextos sociales y económicos específicos» (Gibbs y Eaton, 2014, p. 24 citado en Cepal, 2016).

Frente a la coyuntura abierta por la pandemia del COVID-19 para América Latina, el recorrido realizado permite argumentar que las posibilidades de sobrevivencia y de transitar con las menores consecuencias la crisis socio-sanitaria dependerán del lugar que cada persona ocupe en la estructura social y de los recursos que pueda movilizar a tal fin, así como también de los mecanismos de bienestar y protección social vigentes, incluidas las estrategias ad hoc que cada gobierno articule ante la irrupción del virus.

\section{RECAPITULACIÓN Y COMENTARIOS FINALES}

Para quienes están dispuestos a mirar, la pandemia del COVID-19 se presenta como un espejo que nos devuelve la imagen de una sociedad profundamente diversa y desigual, que deberemos, primero, comprender si pretendemos, posteriormente, modificar. Estas páginas han procurado contribuir a dicho objetivo. Luego de una introducción sobre el problema de las vejeces desiguales en América Latina y la pandemia del COVID-19, se presentaron resumidamente los enfoques del CV y de la EPE. Más tarde, se articularon algunas de sus premisas teóricas en una explicación general sobre la desigualdad existente entre las personas mayores. La figura 1 recapitula este desarrollo:

Cualquier respuesta gubernamental frente a la pandemia que privilegie la opción por la igualdad (o, al menos, por la disminución de las desigualdades) debería comenzar por un diagnóstico certero, atento al reconocimiento de la diversidad de situaciones y problemas que padecen las personas mayores en la región. En esa misma complejidad que asume la realidad social, sería lógico que — desde el punto de vista aquí asumido - actúe el Estado mediante sus políticas públicas y sociales para garantizar el ejercicio los derechos humanos en la vejez. Se espera que este artículo aporte a revisar los marcos analíticos que se utilizan para entender el envejecimiento. Con ese fin, se ha intentado argumentar en torno a los beneficios que presenta una perspectiva longitudinal que considere el curso de vida de las personas y las ventajas y desventajas acumulativas que configuran 
Figura 1. El marco de análisis de las vejeces desiguales

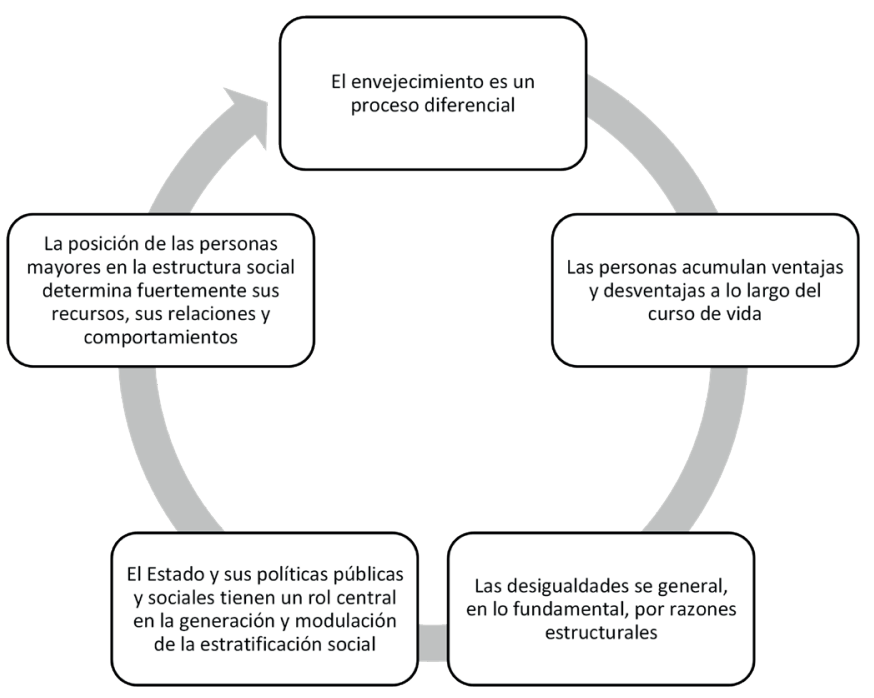

las vejeces desiguales. Al mismo tiempo, se ha insistido en la necesidad de considerar las causas estructurales de estas diferencias, llamando la atención sobre la relación del envejecer de las personas con el Estado y sus políticas públicas y sociales.

En efecto, el punto de vista sugerido permite superar las lecturas transversales y las discusiones que se centran en las diferencias existentes entre los grupos de edad - por ejemplo, aquellas que sugieren más o menos explícitamente que existiría una competencia por los recursos públicos entre los jóvenes y las personas mayores-, poniendo, antes bien, el foco en dilucidar los procesos sociales que subyacen a la aparición de desigualdades a lo largo del a vida y que, en la vejez, terminan por aumentar las diferencias entre las personas. Este ejercicio arroja luz, a su vez, sobre los múltiples y desiguales modos de envejecer en América Latina, poniendo de relieve las condiciones estructurales que influyen en el curso de la vida de los ciudadanos de la región más desigual del mundo.

A modo de cierre, considérense las palabras de Arundhati Roy (2020), cuyo espíritu nos invita a buscar, en el escenario distópico abierto por la pandemia, una oportunidad para reconsiderar en qué tipo de sociedad queremos vivir: 
Históricamente, las pandemias han obligado a los humanos a romper con el pasado e imaginar su mundo de nuevo. Esta no es diferente. Es un portal, una puerta de enlace entre un mundo y el siguiente. Podemos elegir atravesarla, arrastrando los cadáveres de nuestro prejuicio y odio, nuestra avaricia, nuestros bancos de datos e ideas muertas, nuestros ríos muertos y cielos humeantes detrás de nosotros. $\mathrm{O}$ podemos caminar a la ligera, con poco equipaje, listos para imaginar otro mundo. Y listos para luchar por él. (s.p.)

El avance del proceso de inmunización frente al COVID-19 - en curso, todavía no logrado en su totalidad - , redirige los interrogantes hacia la pospandemia. Tras la crisis, será necesario analizar las consecuencias que esta ha dejado a su paso, en especial para los grupos sociales que se encuentran en situaciones de mayor desventaja, y, desde allí, revisar los pactos políticos y sociales que sustentan nuestra vida en común. Se ha comenzado a atisbar algunas pistas sobre el camino a recorrer (Argentina Futura, 2020; Cepal, 2020d; CEPE, 2020; Naciones Unidas, 2020), todas las cuales ponen atención en la necesidad de que esa agenda transformadora se construya a través de un debate abierto y horizontal que encuentre sustento en la perspectiva de los derechos humanos.

\section{REFERENCIAS}

Adelantado, J. et al. (2000). El marco de análisis: las relaciones complejas entre estructura social y políticas sociales. En J. Adelantado, Cambios en el estado de bienestar. Barcelona: Icaria.

Ares Castro-Conde, C. (2018). Envejecimiento y política: un debate politológico. Revista de Estudios Políticos, 179, 171-198. https://doi.org/10.18042/cepc/rep.179.06

Argentina Futura (2020). El futuro después del COVID-19. Buenos Aires: Jefatura de Gabinete de Ministros.

Arundhati, R. (11 de abril de 2020). Rebelión.org. Recuperado de https://rebelion.org/ la-pandemia-es-un-portal/

Bayón, M. C. (2015). La integración excluyente. México: Instituto de Investigaciones Sociales, UNAM. Bonilla Artigas. https://doi.org/10.1080/ 10875549.2016.1141385

Bengtson, V. L. y Warner, S. (eds.) (1999). Handbook of Theories of Aging. Nueva York: Springer Publishing Company.

Bengtson, V. L., Elder, G. H. y Putney, N. M. (2005). The Lifecourse Perspective on Ageing: Linked Lives, Timing and History. En M. L. Johnson (ed.), The 
Cambridge Handbook of Age and Ageing (pp. 493-501). Cambridge: Cambridge University Press. https://doi.org/10.1017/CBO9780511610714.053

Blanco, M. (2011). El enfoque del curso de vida: orígenes y desarrollo. Revista Latinoamericana de Población, 5(8), 5-31. https://doi.org/10.31406/relap2011.v5.i1. n8.1

Bruno, F. y Acevedo Alemán, J. (2016). El enfoque biográfico: la construcción de las trayectorias laborales de los adultos mayores, un fenómeno de análisis social. Kairós Gerontologia, 19(4), 29-47.

Butler, R.N. (1969). Age-ism: Another form of bigotry. The Gerontologist, 9, 243- 246. https://doi.org/10.1093/geront/9.4_Part_1.243

Cepal (2003). Primera Conferencia Regional Intergubernamental sobre Envejecimiento. Recuperado de https://www.cepal.org/es/eventos/conferencia-regional-intergubernamental-envejecimiento

Cepal (2016) Panorama Social de América Latina. Santiago de Chile. Recuperado de https://issuu.com/siproid/docs/s1700567_es/25

Cepal (2020a). Desafios para la protección de las personas mayores y sus derechos frente a la pandemia de COVID-19. Santiago de Chile: Cepal. Recuperado de https://www.cepal.org/sites/default/files/publication/files/46487/S2000723_ es.pdf

Cepal (2020b). Estudio económico de América Latina y el Caribe (LC/PUB.2020/12-P). Santiago de Chile: Cepal. Recuperado de https://repositorio.cepal.org/bitstream/ handle/11362/46070/89/S2000371_es.pdf

Cepal (2020c). La pandemia del COVID-19 profundiza la crisis de los cuidados en América Latina y el Caribe. Santiago de Chile: Cepal.

Cepal (2020d). Pactos políticos y sociales para la igualdad y el desarrollo sostenible en América Latina y el Caribe en la recuperación pos-COVID-19. Santiago de Chile: Cepal.

Cepal y OIT (2018). Coyuntura laboral en América Latina y el Caribe. La inserción laboral de las personas mayores: necesidades y opciones. Santiago de Chile: Naciones Unidas.

CEPE (2020). Pospandemia. 53 políticas públicas para el mundo que viene. Buenos Aires: Universidad Torcuato Di Tella.

Dabove, I. Oddone, J. Perret, C. Pochintesta, P. (2020). Vejez en tiempos de pandemia: una cuestión de derechos. https://doi.org/10.22187/rfd2020n49a10

Dannefer, D. (2003) Cumulative Advantage/Disadvantage and the Life Course: CrossFertilizing Age and Social Science Theory. The Journals of Gerontology. Series B, 58(6), S327-S337. https://doi.org/10.1093/geronb/58.6.S327 
Dannefer, D., y Settersten, R. (2010). The study of the life course: implications for social gerontology. En D. Dannefer y C. Phillipson (eds.), The SAGE Handbook of Social Gerontology (pp. 4-19). Londres: Sage Publications. https://doi. org/10.4135/9781446200933.n1

Díaz Tendero Bollain, A. (2016). La teoría de la economía política del envejecimiento. Un nuevo enfoque para la gerontología social en México. México: El Colegio de la Frontera Norte.

Estes, C. y Di Carlo, N. (2020). The Right to Work and the Right to Retire: A Political Economy Perspective on Precarity. Generations: Journal of the American Society on Aging, 43(3), The Future of Work and Older Workers (Fall 2019), 29-34.

Feixa, C. (1996). Antropología de las edades. En J. Prat y A. Martínez (eds.), Ensayos de Antropología Cultural. Homenaje a Claudio Esteva-Fabregat. Barcelona: Ariel.

Ferraro, K.F. y Shippee, T.P. (2009). Aging and Cumulative Inequality: How Does Inequality Get Under the Skin? The Gerontologist, 49(3), 333-343. https://doi. org/10.1093/geront/gnp034

Gastrón, L., Oddone, J. y Lynch, G. (2011). Ganancias y pérdidas a lo largo de la vida. En J.A. Yuni (comp.), La vejez en el curso de la vida. Córdoba: Encuentro Grupo Editor.

Gastrón, L. y Oddone, M. J. (2008). Reflexiones en torno al tiempo y el paradigma del curso de vida. Perspectivas en Psicología, 5(2), 1-9.

Helpage-International (2019). Salud y bienestar, ¿cuál es la percepción de las personas mayores? En países de ingresos medianos y bajos. Londres: HelpAge-international.

Huenchan Navarro, S. (2004). Políticas sobre vejez en América Latina: elementos para su análisis y tendencias generales. Notas de Población, 78, 155-182.

Huenchuan, S. (2018) (ed.). Envejecimiento, personas mayores y Agenda 2030 para el Desarrollo Sostenible: perspectiva regional y de derechos humanos. Santiago de Chile: Cepal. https://doi.org/10.18356/19532890-es

Iacub, R. (2002). La postgerontología: hacia un renovado estudio de la gerontología. Revista Latinoamericana de Psicología, 34(1-2), 155-157.

Kending, H., Nazroo, J. (2016). Life Course Influences on Inequalities in Later Life: Comparative Perspectives. Population Ageing, 9, 1-7. https://doi.org/10.1007/ s12062-015-9138-7

Knopoff, R. y Oddone, J. (1991) (comps.). Dimensiones de la vejez en la sociedad argentina. Buenos Aires: Centro de Editor de América Latina. 
Korfeld-Matte. United Nations Human Rights. Office of the High Commissioner (2020). «Unacceptable» - UN expert urges better protection of older persons facing the highest risk of the COVID-19 pandemic. Recuperado de https://www.ohchr.org/ EN/NewsEvents/Pages/DisplayNews.aspx?NewsID=25748\&LangID=E

Lalive D’Epinay, C. Bickel, J.F., Cavalli, S. Spini, D. (2011). El curso de la vida: emergencia de un paradigma interdisciplinario. En J.A. Yuni (2011) (comp.), La vejez en el curso de la vida. Córdoba. Encuentro Grupo Editor.

Lesson, G. (2013). The demographics of population ageing in Latin America, and the Caribbean and the Iberian Peninsula, 1950- 2050. En V. Montes de Oca (ed.), Envejecimiento en América Latina y el Caribe. Enfoques en investigación $y$ docencia de la Red Latinoamericana de Investigación en Envejecimiento (LARNA). México: UNAM.

Ludi, M. d. C. (coord.) (2018). Familia y vejez. Configuraciones familiares y procesos de envejecimiento en el actual contexto. Ciudad Autónoma de Buenos Aires: Espacio Editorial.

Lynch, G. (2015). Modelos del Curso de la Vida: transformaciones y continuidades. XI Jornadas de Sociología. Facultad de Ciencias Sociales, Universidad de Buenos Aires.

Manes, R., Carballo, B., Cejas, R., Machado, E., Prins, S., Savino D. y Wood, S. (2016). Vejeces desiguales. Un análisis desde el enfoque de derechos de las personas mayores. Revista Margen de Trabajo Social y Ciencias Sociales.

Manes, R., Garmendia, C. y Danel, P. (2020). Envejecimiento y vejeces: aproximaciones conceptuales desde la decolonialidad. En C. B. Tello y P. Danel (coords.), Decolonialidad, identidades divergentes e intervenciones. La Plata: Facultad de Trabajo Social, UNLP.

Naciones Unidas (2020). Respuesta integral de las Naciones Unidas a la COVID-19: salvar vidas, proteger a las sociedades, recuperarse mejor. Recuperado de https://www.un.org/sites/un2.un.org/files/comprehensive_response_to_covid19 spanish.pdf

O'Rand, A.M (2006). Stratification and the Life Course. Life Course Capital, Life Course Risks, and Social Inequality. En R. H. Binstock y L. K. George (eds.), Handbook of Aging and the Social Sciences (sexta ed.) Elsevier. https://doi. org/10.1016/B9-78-012088-3/88250-0122

Oddone, J. (2014). El desafio de la diversidad en el envejecimiento en América Latina. Voces en el Fénix. Universidad de Buenos Aires.

Oddone, J. (2018). Condiciones de vida de las personas mayores. En J. Piovani y A. Salvia, La Argentina en el siglo XXI. Cómo somos, vivimos y convivimos en una 
sociedad desigual: Encuesta Nacional sobre la Estructura Social. Buenos Aires: Siglo XXI.

OISS e IMSERSO (2006). Situación, necesidades y demandas de las personas mayores en los países del Cono Sur. Apuntes para un diagnóstico. Recuperado de http:// envejecimiento.csic.es/documentos/documentos/oiss-brasil-01.pdf

OPS y OMS (2021). Construir salud a lo largo curso de vida. Conceptos, implicaciones y aplicación en la salud pública. Recuperado de https://iris.paho.org/bitstream/ handle/10665.2/53368/9789275323021_spa.pdf?sequence=3\&isAllowed=y

Ossorio, P. (2017). Envejecer en el siglo XXI en América Latina. S.L. Recuperado de https://biblio.flacsoandes.edu.ec/catalog/resGet.php?resId=24838

Pérez Díaz, J. (2016). El temor al envejecimiento demográfico. En S. Ezquerra, M. Pérez Salanova, M. Pla y J. Subirats (eds.), Edades en transición. Envejecer en el siglo XXI. Edades, condiciones de vida, participación e incorporación tecnológica en el cambio de época. Barcelona: Ariel.

Piña Morán, M. (2010). Matriz de intervención en gerontología social. RUMBOS TS, $\mathrm{V}(5)$.

Ramos Bonilla, G. y Tirado Ratto, E. (2019). Hasta que el cuerpo aguante. Trayectorias, rutinas y motivaciones laborales de trabajadores adultos mayores de la ciudad de Lima, Perú. Bulletin de l'Institut Français d'Études Andines, 48(3), 381-404. https://doi.org/10.4000/bifea.11078

Redondo, N. (1999). Ancianidad y pobreza. Una investigación en sectores populares. Buenos Aires: Hvmanitas.

Rossel, C. y Filgueira F. (2015). Vejez. En S. Cecchini, F. Filgueira, R. Martínez y C. Rossel (eds.), Instrumentos de protección social. Caminos latinoamericanos hacia la universalización. Santiago de Chile. Cepal. Recuperado de https://repositorio.cepal.org/bitstream/handle/11362/39671/ S1500279_es.pdf? sequence $=1 \&$ isAllowed $=\mathrm{y}$

Roy, A. (4 de abril de 2020). La pandemia es un portal. Recuperado de http://comunizar. com.ar/arundhati-roy-la-pandemia-portal/

Saraví, G. (2020). Acumulación de desventajas en América Latina: aportes y desafíos para el estudio de la desigualdad. RELAP - Revista Latinoamericana de Población, 14(27), 228-256 http://doi.org/10.31406/relap2020.v14.i12.n27.7

Saravi, G. (ed.) (2007). De la pobreza a la exclusión. Continuidades y rupturas de la cuestión social en América Latina. México: Prometeo / CIESAS.

Subirats, J. (2016). Cambio de época y personas mayores. Una concepción de ciudadanía abierta e inclusiva. En S. Ezquerra, M. Pérez Salanova, M. Pla y J. Subirats (eds.), Edades en transición. Envejecer en el siglo XXI. Edades, condiciones de 
vida, participación e incorporación tecnológica en el cambio de época. Barcelona: Ariel.

United Nations, Department of Economic and Social Affairs, Population Division (DESA Population) (2019). World Population Prospects 2019: Ten Key Findings.

Walker, A. (2006). Aging and Politics: An International Perspective, En R. H. Binstock y L. K. George (eds.) (sexta ed.). Handbook of the Aging and the Social Sciences. Amsterdam: Academic Press.

Walker, A. (2009). Ageing and Generational Politics, En Flinders M., Gamble, A., Hay, C. y Kenny, M. (eds.), The Oxford Handbook of British Politics. Oxford Handbooks. https://doi.org/10.1093/oxfordhb/9780199230952.003.0036

Yuni, J. (2019). Prólogo. En P. Danel y M. Navarro (comps.), La gerontología será feminista. Buenos Aires: Fundación La Hendija.

Yuni, J.A. Integraciones metateóricas en el paradigma del curso de la vida. En J.A. Yuni (2011) (comp.), La vejez en el curso de la vida. Córdoba: Encuentro Grupo Editor. 